\title{
Cerebrolysin as a New Treatment Option for Post- Stroke Spasticity: Patient and Physician Perspectives
}

Nataliia Chemer · Volodymyr Bilanovskyi

Received: October 9, 2018 / Published online: February 18, 2019

(C) The Author(s) 2019

\begin{abstract}
Introduction: Recent studies show that spasticity occurs in $20-30 \%$ of all stroke victims, especially in younger patients. Botulinum toxin is a very effective treatment; however, it is not reimbursed in the Ukraine, thus leaving patients untreated and unable to use the paralyzed limb. Finally, this might result in longterm disability with economic consequences and negative impact on the psychological state of patients. Therefore, alternative and affordable treatment options are of high interest. This case report describes the therapeutic experience with Cerebrolysin on post-stroke spasticity in a chronic stroke patient from both the investigator's and the patient's perspective.

Methods: Cerebrolysin was administered for 30 days at a daily dose of $10 \mathrm{~mL}$ by intramuscular (IM) injections into the spastic limb of a 56-year-old chronic stroke patient. Therapeutic effects were assessed by the Modified Ashworth
\end{abstract}

Enhanced digital features To view enhanced digital features for this article, go to https://doi.org/10.6084/ m9.figshare.7624022.

N. Chemer $(\varangle)$

Head of Stroke Unit, Kyiv Regional Clinical

Hospital, Kiev, Ukraine

e-mail: chemerko@ukr.net

V. Bilanovskyi

Kiev, Ukraine
Scale (MAS), the mRS, and manual muscle testing (MMT).

Results: After completing the treatment course with Cerebrolysin, spasticity-related outcome measures like MMT had improved by $70 \%$ and the MAS by 2 points. The patient reported a beneficial effect on mood and motivation.

Conclusion: Intramuscular treatment with Cerebrolysin of post-stroke spasticity was safe and effective in this patient. The experiences observed in this patient are in line with the findings of a larger cohort study treated in the same way in the Philippines.

Keywords: Cerebrolysin; Post-stroke spasticity; Rehabilitation

\section{PATIENT'S EXPERIENCE}

I had a stroke 5 years ago. It was a severe hemorrhagic stroke. During the first 3 months I was not very well aware of my situation and do not remember what has happened then. I was able to move independently and to walk with support after 3 months, but my left hand did not function at all. I was determined to improve the situation! Indeed, I made progress in walking by reducing spasticity in the leg muscles, but the hand remained immovable. A few months later, I suffered from a deep achy pain in the paralyzed hand and in the leg, like muscle pain after an intense physical training. At the beginning, 
this pain appeared sporadically, but later it became my permanent companion. It was associated with spasticity as my doctor explained to me. The spasticity in my leg actually helped to straighten it, and I could walk more confidently with a stick without support. The situation with the hand was different: the fingers were bent into a fist and I could not straighten them, only with assistance and considerable effort. However, I did not give up and tried to continue doing exercises as it was advised earlier in the clinic. Over time the situation worsened and it became almost impossible to do exercises with my left hand and leg. Moreover, the pain became permanent. I consulted my doctor and he recommended botulinum toxin injections as a possible solution to my problem. I am not the person who gives up easily, so I agreed immediately despite the high price. At the beginning, we decided to try treatment only on the left hand. To my disappointment, I did not notice any effect of the procedure: my fist remained clenched and my wife helped me to unbend my hand to get dressed. Since it was already 2 years after the stroke, I began to despond and my hopes for recovery were disappearing. Nevertheless, I started to work again as car mechanic, which gave me additional opportunity to network with people and did not allow me to become depressed. My family was very supportive as well. Once I met an employee with the same problem at a gas station-his left hand was paralyzed, but he was able to use it to perform his duty. I asked him how he was living with this problem. He advised me to continue exercises despite everything and try to use the affected hand as much as possible. It incredibly supported and inspired me. Just a few months later I learned from my doctor about a possibility to take part in a clinical research for patients with a similar problem. The doctor explained that Cerebrolysin's ability to reduce spasticity in paralyzed limbs will be explored. One month later, after daily injections, I was able to dress myself and to raise my hand, and it became possible to unclench the fingers with assistance, which was previously impossible. I continued my exercises and my achy pain disappeared. At the end of the treatment course, I could walk without a walking stick for long distances! I continued to use my left hand as often as possible: when repairing cars, I tried to hold a wrench with my left hand, I tried to turn off lights and to take a shower without any assistance. I felt a sensation in my left hand, which was absent before; "a shape of an object in my hand" sensation returned, and coordination and accuracy of movements improved. I was especially happy that the effect was persistent and improved over time. It inspired and stimulated me to continue exercises. Now I can unbend the fingers, use my left hand at work and at home, and drive a car. Ten months have passed since the treatment course with Cerebrolysin. I still feel the effects of the treatment and plan to repeat the course.

\section{PHYSICIAN'S PERSPECTIVE}

The history of this patient is unusual for two reasons. First, the treatment of spasticity with Cerebrolysin is a new method, initially described by Martinez [2]. Cerebrolysin has not been used previously for spasticity treatment in our practice. Secondly, it is rare that a patient remains highly motivated and continues exercises with such persistence to restore his motor functions. Usually, patients are more likely to rely on rehabilitation specialists and do not continue exercising at home properly after discharge from the clinic, and they often lose the hope for recovery. Spasticity is an important factor influencing rehabilitation progress negatively. Post-stroke spasticity causes painful sensations and the inability to use a paralyzed limb in everyday routine, which leads to the demotivation of the patient. I was intrigued to use Cerebrolysin in spasticity treatment for two reasons:

1. Cerebrolysin intramuscular administration into a paralyzed spastic limb at a dosage of $10 \mathrm{ml}$ daily over 1 month was shown to be effective and reduced spasticity symptoms in the long term [2]. Furthermore, it can be combined with physical exercises within a standard rehabilitation program [1].

2. Additionally, its perceived antidepressant effect should improve the patient's general 
mood, increases motivation for physical activity, and improves cognitive function and motivation, which altogether may results in a significant positive outcome.

The patient of this case study was male, born in 1959, and suffered from stroke on June 24th, 2013 with spasticity diagnosed on July 4th, 2013. The spasticity-related rehabilitation program was initiated on September 12th, 2017; at that time, the patient had an NIHSS score of 6 . At follow-up 180 days later, the NIHSS score was 5 and the distal motor function score decreased from 2 to $0-1$. In the manual muscle testing, the patient improved by approximately $70 \%$ and by 1-2 points in the Modified Ashworth Scale.

Taking into account the small number of centers and insufficient number of specialists providing quality rehabilitative treatment in our country, the access to this type of care is limited. Therefore, treatment of spasticity with Cerebrolysin is an encouraging approach as it is easy to use, accessible, and affordable, andmost important-safe to use in outpatient care. However, a key factor for success in post-stroke spasticity management is the motivation of the patient and people supporting him/her to continue rehabilitation. If a rehabilitation program has a clear goal and achievable tasks that go beyond daily routine and allows patients to participate in social life again, it will make therapy more successful!

\section{ACKNOWLEDGEMENTS}

Funding. No funding or sponsorship was received for this study or publication of this article.
Authorship. All named authors meet the International Committee of Medical Journal Editors (ICMJE) criteria for authorship for this article, take responsibility for the integrity of the work as a whole, and have given their approval for this version to be published.

Disclosures. Chemer Nataliia and Bilanovskyi Volodymyr have nothing to disclose.

Compliance with Ethics Guidelines. This article does not contain any studies with human participants or animals performed by any of the authors.

Open Access. This article is distributed under the terms of the Creative Commons Attribution-NonCommercial 4.0 International License (http://creativecommons.org/licenses/ by-nc/4.0/), which permits any noncommercial use, distribution, and reproduction in any medium, provided you give appropriate credit to the original author(s) and the source, provide a link to the Creative Commons license, and indicate if changes were made.

\section{REFERENCES}

1. Martinez RM. Efficacy of Cerebrolysin in reduction of spasticity during stroke rehabilitation. J Med Life. 2017;10:161-6.

2. Muresanu DF, Heiss WD, Hoemberg V, et al. Cerebrolysin and recovery after stroke (CARS): a randomized, placebo-controlled, double-blind, multicenter trial. Stroke. 2016;47:151-9. 